\title{
Dep VII. Aerzte-Congress zu Kasan
}

(gestiftet zum Andenken Pirogoff's), tagte vom 28. April bis õ. Mai (a. St.) d. J.

In der ophthalmologischen Abteilung waren folgende Themata zur Diskussion gestellt:

Trachom,

Katarakt,

Subkonjunktivale Injektionen in therapeutischer Beziehung. In Folge Zeitmangels kamen jedoch nicht alle Fragen zur Be-

sprechung.

Der Vorsitzende, Prof. Adamück, begrüsste die An $\Lambda$ vesenden und gab zunächst in längerer Kede eine umfassende Zusammenstellung der geographischen Verbreitung des Trachoms, sprach dann über die bezüglich Jiassen- und individueller Disposition zu der Krankheit be-kannten Thatsachen und erklärte sich als strenger Dualist bezüglich der Unterscheidung von Trachom und Follikularis als besonderer Krank-heiten. Des "weiteren ging er auf die üblichen Behandlungsmethoden des Trachoms (medikamentös-antiseptische, mechanische, operative) ein,, ohne im einzelnen neue therapeutische Vorschläge zu machen.

Zur Bekämpfung des Trachoms als Volksseuche empfiehlt Redner:

Aufstellung einer möglichst genauen Statistik über die Zahl der vorhandenen Trachomkranken; Einrichtung einer möglichst grossen Zahl von Ambulatorien resp. Heilaństalten in den Seucheherden;

Ausbildung praktischer Aerzte in der Behandlung der Granu-lose (lurch zweimonatliche Kurse an grossen Kliniken;

Verbreitung von popular geschriebenen Brochüren über die Art und Ausbreitungsweise der Granulose;

$\delta$. Uebertragung der Sorge für die Bekämpfung der Krankheit an

den Verein „des roten Kreuzes”. Dr. Walter-Odessa bekennt, dass er Trachom und Follikularis für verschiedene Abstufungen ein und derselben Krankheit betrachtet

212

Sitzimgsberichte.

und nur therapeutiscb einen Unterschied zwischen schweren und leichten Formen der Erkrankung mache.

In der Behandlung Yerwirft W. die scharfen Beizungen bei der sogenannten Conj. follicularis, bei den schweren Formen gebraucht er Arg. nitricum, Cupr. sulf. und zuweilen den Knapp'schen Roller. Von anderen Eingriffen sah er wenig Erfolg.

Hierauf wurde ein von einer besonderen Commission ausgearbeiteter Entwurf eines Schemas für eine allgemeine Trachomstatistik der Ver-sammlung unterbreitet und von dei·selben angenommen.

Man einigte sich dahin, dass in diesei- Statistik nur alle allge-mein als Granulose angesehenen Fälle Aufnahme finden sollen, ferner alle suspekten Fälle (nach besonderen festgesetzten Merkzeichen zu diagnostizieren); dagegen sollen typische Fälle von Follicularis ausge-schlossen sein. 
In dem Programm des nächsten Congresses soil die Besprechung der Behandlung und Prophylaxe des Trachoms nochmals aufgenommen werden. Hierauf spricht

Dr. Rymovitsch-Kasan. Beitrag zur Aetiologie der akuten ansteckenden Bindehautentzündun g. R. konstatierte in 16 untersuchten Fallen das Vorhandensein der K och- Weeks'schen Bazillen, bringt im übrigen nichts wesentlich Neues.

Dr. Rudin-Tula: Die Wirkung der russischen Dampfbade-stube auf den Blutumlauf in der Netzhaut des Auges.

R. ist zu keinen einwandfreien Resultaten gekoramen, glaubt jedoch gefunden zu haben, dass unter dem Einfhiss der r. I), nicht eine Hyperämie, (Fialkowski? Frei) sondern eine arterielle Anämie zu stande kommt, sodass ein günstiger Einfluss .auf Ent-zündungsprozesse in der Netzhaut anzunehnien ist, zumal unter Berüek-sichtigung der durch die enorme Transpiration beschleunigten Auf-saugung pathologischer Exsudate.

Dr. Walter-Odessa: „Die offene Behandlung der Wunden nach Operationen am Augapfel.” $W$. hat mit der völlig offenen $\Lambda$ Vundbehandlung nach Hjort gute Erfahrungen gemacht (Beobachtung von 60 Fallen) ist jedoch nicht für prinzipielle Anwendung derselben, weil sie bei unver-ständigen Patienten zu gefahrvoll ist.

Er giebt den Behandlungsmethoden von Czermak, Praun etc., (kein Verband des Auges direkt, doch Schutz des operierten Oculus durch ein Gitter oder Netz) für viele Fälle den Vorzug. Für Fälle von Ciliarreizung, Drvcksteigerung und Glaskörper-vorfall will er den Bindenverband auch weiterhin nicht missen.

Die Vorträge des Dr. Egoroff: „Operation bei Ektropium des Auges” und des Dr. Agababoff: „Angaben über die Cataract” kamen ínfolge Zeitmangels nicht mehr zur Verlesung.

Zum Scbluss sei noch erwähnt, dass Dr. Walter-Odessa der Versammlung folgende Vorschläge unterbreitete:

-1. Die Bildung einer allrussischen ophthalmologischen Gesell-schaft nach Master der Heidelberger.

Sitzungsberichte.

213

Den Versuch, geeignete Massregeln zu ergreifen, um der Er-blindugg in Russiand nacli

Möglichkeit zu steuern.

Die Organisierung einer ärztlichen Aufsicht über Schüler und Arbeiter.

Einreichung von Gesuehen an die betreffenden Behörden und mentsprechende Publikationen in der Presse zum Zweck der praktischen Durchführung der unter 2 und 3 vorgeschlagenen Massnahmen.

Dr. A gab ab of f-Kasau.

Soeiété belg·e d'ophthalmolog·ie.

Sitzung vom April 1899.

Nuel und Benoit: Die Absorption des Humor aqueus immenschlichenAuge.

Dass beim Menschen vornehmlich die Iris den Weg zur Aus-scheidung des Humor aqueus darstellt, fanden die beiden Autoren in z $\lambda$ vei Experimenten, die sie an menschlichen Augen vor deren Enu-cleation vornalimen; sie injizierten 2 Tropfen lauwarrner und aseptischer chinesischer Tusclie in der Gegend cles Augenaequators in das Corpus vitreum 212 Stunden vor der Operation des ersten Auges: Die chinesi-sche Tusche drang in die Vorderpartieen des Auges und erschien in der vorderen Kammer. Fast ausschliesslich durch die Iris drang die Tusche ein, verbreitete sich entlang den Gefässwandungen, niemalsaber im Lumen derselben. 
Der Farbstoff durchdringt die Iris durch die Krypten der vor-mderen Lage, besonders durch die der Irisbasis. üebergänge in die Sclera wurden nicht beobachtet.

In der líöhe des Schlemm'schen Canals ist der Farbstoff nicht durchgetreten. - Er dringt ausserdem in das Corpus ciliare ein, ent-entlang den Processus ciliares und durchtränkt die Gefässwandungen.

In der Retina, der Chorioidea und dem Nervus opticus findet sich keine Spur von der Tusche. Im zweiten Auge (Injection 4 Stunden vor der Enucleatiou) ist im Verlauf von 10 Minuten der Farbstoff in grosser Menge in die vor- $<$ iere Kammer eingedrungen, sodass diese ganz schwarz erscheint. - Sonst linden sich die gleichen Erscheinungen, wie am ersten Auge.

Die Ausscheidung durch den Nervus opticus, die man beim Glau-koma posterius sehr hoch glaubte anschlagen zu niüssen, ist für den Menschen durch die genannten Autoren nicht bestä,tigt worden, aber sie existiert in reichlicherem Masse beim Kauinchen.

Van Duyse: Deber Anophthalmus congenitus.

D. Die anatomische $\Gamma$ ntersuchung eines Falles von $\mathrm{d} \theta]$ )pels(v́tigern Anophthalmus congenitus und das Studium der vorher publizierten Beobachtungen darüber haben den Autor zu der Ueberzeugung geführt

dass die Mehrzahl der sehon aufgezählten Fälle von Anophthalmus nur einen hohen Grad von Mikrophthalmus repräsentiert.

Im Falle des Autors setzt sich das Augenknötchen ( $1 \mathrm{X} \delta \mathrm{wm})$ aus zwei Abkommlingen des Mesoblast zusammen: aus der Sklera und 